\title{
REABILITAÇÃO ESTÉTICA ANTERIOR COM FACETAS E COROAS CERÂMICAS: RELATO DE CASO CLÍNICO
}

\author{
ANTERIOR ESTHETIC REHABILITATION WITH PORCELAIN VENEERS \\ AND CERAMIC CROWNS: A CASE REPORT
}

\author{
William BARNABÉ1; Marco Aurélio de CARVALHO²; Geovani Vitorino BORGES³; Yuri A. 0. BARBOSA3; Célio Umberto ARAÚJ0; \\ Priscilla Cardoso LAZARI-CARVALHO² \\ 1 - Professor Adjunto da área de Prótese Dentária da Faculdade de Odontologia da Universidade Federal de Goiás (FO-UFG). \\ 2 - Professor da área de Dentística da Faculdade de Odontologia do Centro Universitário de Anápolis - UniEVANGÉLICA. \\ 3 - Cirurgião-Dentista. \\ 4 - Professora da área de Prótese Dentária da Faculdade de Odontologia do Centro Universitário de Anápolis - UniEVANGÉLICA.
}

\section{RESUMO}

Objetivo: Este trabalho tem como objetivo apresentar um caso clínico de reabilitação estética anterior (centrais e laterais anterossuperiores) associando facetas e coroas cerâmicas cimentadas com diferentes técnicas (cimento resinoso e resina composta aquecida) com a finalidade de reproduzir as características dos dentes naturais. Material e Métodos: Paciente A.M.A., gênero feminino, 47 anos, compareceu à clínica odontológica da Faculdade de Odontologia da Universidade Federal de Goiás (UFG), relatando insatisfação com a estética do seu sorriso, diferenças no formato e cor de seus dentes. Ao exame clínico intrabucal verificou-se uma coroa total provisória do dente 22, e o mau posicionamento do dente 12 em relação aos adjacentes. Foi indicada cirurgia periodontal para aumento de coroa clínica, clareamento dental, duas facetas cerâmicas nos incisivos centrais e duas coroas cerâmicas nos incisivos laterais superiores. Após a cirurgia periodontal e clareamento, foi realizado o refinamento dos preparos para coroa total e preparos para faceta cerâmica. O material de escolha para as restaurações foi o dissilicato de lítio devido sua grande capacidade de mimetizar o dente natural. As coroas foram cimentadas com isolamento relativo do campo operatório e cimento resinoso dual. As facetas foram cimentadas com isolamento absoluto e resina composta aquecida. Resultados: Após 15 dias da cimentação a paciente retornou à clínica onde se observou saúde periodontal e perfeita adaptação das restaurações indiretas. Conclusão: Obteve-se excelente resultado estético associando diferentes tipos de restaurações e técnicas de cimentação adesiva e a paciente se mostrou confortável com as diferentes abordagens e satisfeita com o resultado estético do tratamento.

Palavras-chave: Facetas dentárias; Coroas; Cimentos dentários; Colagem dentária; Reabilitação bucal.

\section{INTRODUÇÃO}

Atualmente, as restaurações cerâmicas adesivas, como as coroas totais ou facetas, apresentam um conceito restaurador previsível em termos de longevidade clínica, resposta periodontal e resposta dos pacientes ${ }^{1}$. Devido ao seu alto apelo estético, biocompatibilidade comprovada e previsibilidade em longo prazo, as facetas totalmente em cerâmica tornaram-se um dos materiais de eleição nas reabilitações em dentes anteriores².

O sucesso em longo prazo das restaurações adesivas é determinado pelas propriedades do material de escolha, resistência à fadiga dos sistemas de cerâmica, cimento adesivo/agente cimentante utilizados, bem como os preparos realizados para receber as restaurações ${ }^{2}$. A odontologia restauradora atual trabalha com o conceito dos procedimentos minimamente invasivos em qualquer tipo de reabilitação oral, evitando desgastes desnecessários do elemento dental. No entanto, sabe-se que as restaurações indiretas requerem certa quantidade de desgaste garantindo um término definido no preparo, que quando planejado e controlado, pode ser conservador e efetivo a fim de garantir a estética e longevidade adequadas ${ }^{3}$. Fica evidente então, o equilíbrio entre o conservadorismo no preparo dentário, a criação do eixo de inserção, término cervical e quantidade de desgaste, para garantir a perfeita adaptação da peça juntamente com a espessura adequada da restauração ${ }^{3}$.

As facetas cerâmicas se caracterizam pelo recobrimento da face vestibular do dente por um material restaurador, podendo ser confeccionada pela técnica direta ou indireta ${ }^{4-6}$. As facetas indiretas são indicadas quando surgirem problemas dentais quanto à forma, posição, simetria, textura superficial e $\operatorname{cor}^{7-9}$. No entanto, nem sempre é possível restaurar os dentes com facetas, visto que muitos deles já apresentam grandes lesões cariosas, fraturas e/ou restaurações pré-existentes com redução significativa de estrutura dentária, o que implica na necessidade de confecção de coroas totais. Seu sucesso clínico depende da qualidade do preparo, assim como da qualidade e quantidade de remanescente coronário, técnicas adesivas e cuidados do paciente ${ }^{10}$.

Além disso, a escolha do agente cimentante e o controle de umidade durante a cimentação pode afetar o sucesso das restaurações cerâmicas ${ }^{11}$. O isolamento do campo operatório deve ser sempre executado para que haja um efetivo controle de 
umidade, seja ele relativo (sugador e roletes de algodão) ou absoluto (lençol de borracha e grampos) ${ }^{12}$.

Diante desse contexto, este relato de caso tem por objetivo apresentar um caso clínico de reabilitação estética dos dentes anteriores, descrevendo protocolo de confecção dos preparos, moldagem e cimentação de facetas e coroas totais em cerâmica.

\section{RELATO DE CASO CLÍNICO}

Paciente A.M.A., gênero feminino, 47 anos, compareceu à clínica odontológica da Faculdade de Odontologia da Universidade Federal de Goiás (UFG), relatando insatisfação com a estética do seu sorriso, diferenças no formato e cor de seus dentes (Figura 1). Ao exame clínico intrabucal verificou-se uma coroa total provisória do dente 22, e o mau posicionamento do dente 12 em relação aos adjacentes.

Antes de iniciar o tratamento, foi realizada a documentação fotográfica e confecção dos modelos de gesso para diagnóstico e planejamento. Com as fotografias, radiografias, modelos e avaliação clínica, optou-se por realizar cirurgia periodontal de aumento de coroa clínica nos dentes 11, 12, 21 e 22 para regularizar a margem gengival dos dentes anteriores previamente ao tratamento restaurador (Figura 2 e 3). Após o período de cicatrização do tecido gengival foi proposto o tratamento restaurador associando coroas totais (nos dentes 12 e 22) e facetas (nos dentes 11 e 21) em porcelana após clareamento dental. A paciente assinou o termo de consentimento livre e esclarecido e termo de autorização de tratamento.

O clareamento dentário foi realizado associando a técnica de consultório e a técnica caseira. O clareamento de consultório foi feito usando peróxido de hidrogênio à 35\% (Whiteness HP Maxx FGM, Joinville, SC, Brasil), em 3 aplicações de 20 minutos. Já para o clareamento caseiro foram confeccionadas moldeiras de silicone para aplicação de gel clareador (Whiteness Simple $22 \%$, FGM, Joinville, SC, Brasil). A paciente foi orientada a usar o produto no protocolo de 01 hora por dia, durante o período de 15 dias. A cor inicial foi A1 segundo a escala Vita Classic (Vita, Zahnfabrik, Sackingen, Alemanha) e ao final do procedimento a cor obtida foi B1. (Figura 4)

Foi realizada então uma moldagem de estudo com silicona de condensação (Perfil, Coltene) para realização do enceramento diagnóstico. Com o enceramento pronto e aprovado, foi feita a moldagem do modelo encerado com silicona de condensação e obtenção do guia de mock-up para inserção da resina bisacrílica. Foram realizados testes fonéticos, observou-se o suporte labial e a anatomia dos dentes, que foram aprovados pela paciente.

Os dentes foram preparados para receberem facetas e coroas totais. Os dentes 12 e 22 já apresentavam preparo para coroa total sendo então realizado apenas seu refinamento com ponta diamantada no 4138 (KG Sorensen, Brasil) (Figura 5). Os dentes 11 e 21 foram preparados para receberem facetas com preparos minimamente invasivos. Foi pontas diamantadas de granulações finas e ultrafinas, nํㅜ 3216, 2200 e 4138 (KG Sorensen, Brasil), consistindo na remoção dos ângulos vivos e áreas retentivas, para que houvesse eixo de inserção e adaptação das facetas cerâmicas, porém sempre mantidos preparos em nível de esmalte. A partir do modelo do enceramento diagnóstico foram confeccionadas novas restaurações provisórias, de modo que as mesmas teriam as mesmas medidas da restauração final (altura, largura

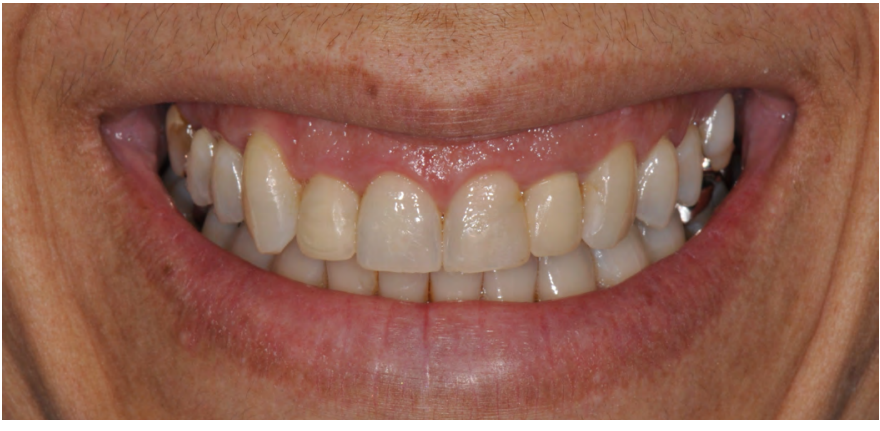

Figura 1 - Foto inicial do sorriso da paciente.

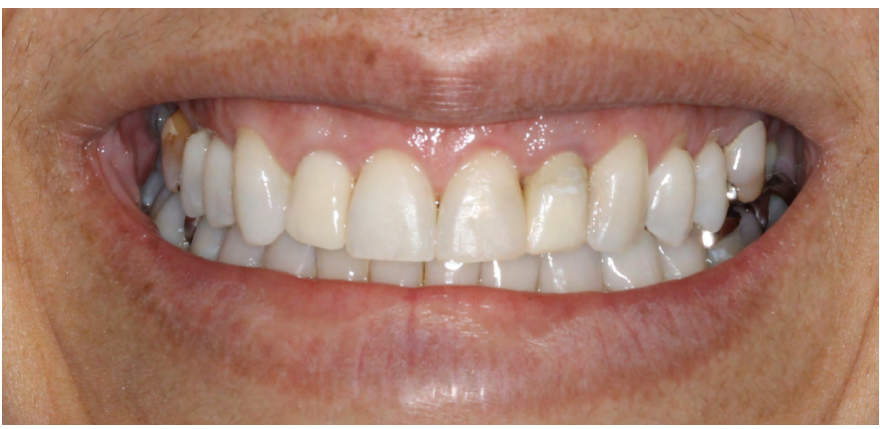

Figura 2 - Foto após a cirurgia periodontal de aumento de coroa clínica.

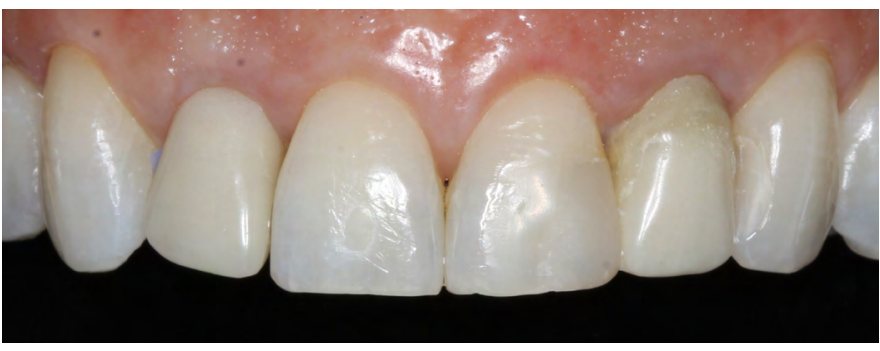

Figura 3 - Foto aproximada dos incisivos superiores apresentando coroas provisórias em resina acrílica dos dentes 12 e 22, e restaurações em resina composta nos dentes 11 e 21.

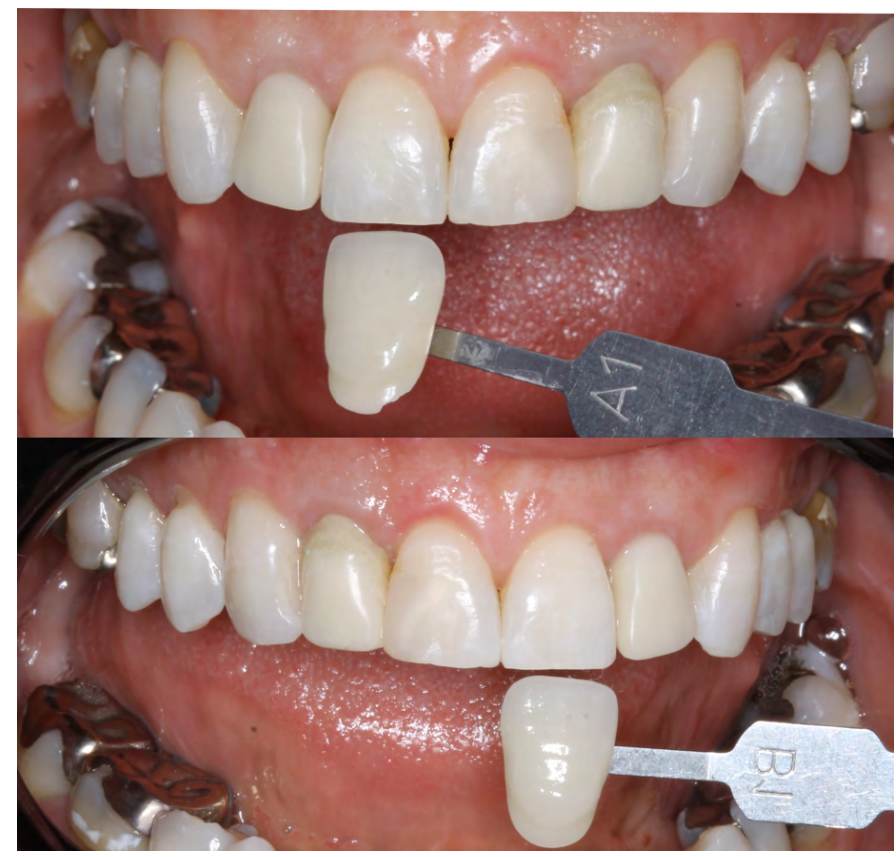

Figura 4 - A- Aspecto inicial antes do clareamento; B- Aspecto final depois do clareamento 
e forma). Ambas foram cimentadas com cimento provisório à base de óxido de zinco sem eugenol, Temp Bond NE (Kerr, Alemanha) (Figura 6).

Após o condicionamento do tecido gengival com as novas restaurações provisórias, os preparos foram moldados com silicona de adição (Variotime Light Flow, Kulzer) pela técnica da dupla impressão, utilizando material leve e pesado. Foi realizada a inspeção visual das peças cerâmicas (Figura 7), seguida da prova seca para que fosse confirmada a adaptação no término cervical, proximal e interoclusal (Figura 8). Para a avaliação da cor, pela interação das restaurações com o substrato dental, realizou-se a prova úmida, com água. Uma vez observado o valor da restauração na prova úmida, seleciona-se a cor do agente cimentante. No presente caso optou-se pela cimentação das facetas com resina composta termomodificada (RTM) e a cimentação das coroas com cimento resinoso dual. Para a seleção de cor da resina indicada para cimentação deve se observar se existe a necessidade de abaixar, manter ou aumentar o valor da cor da restauração. No presente caso clínico, mantivemos a cor do substrato, utilizando uma resina na cor A1 (Z100, 3M ESPE).

A cimentação foi realizada de forma distinta para os diferentes tipos de restaurações: as facetas foram cimentadas sob isolamento absoluto e com resina composta aquecida, enquanto as coroas totais foram cimentas sob isolamento relativo com cimento resinoso dual. A resina composta A1 (Z100, 3M ESPE) foi aquecida em dispositivo próprio (Calset, Addent) para a cimentação das facetas. O cimento resinoso Variolink Esthetic DC Neutral (Ivoclar Vivadent) foi utilizado para a cimentação adesiva das coroas.

Iniciou-se a cimentação pelas facetas (11 e 21), sob isolamento absoluto. Simultaneamente foram feitos os preparos das superfícies do dente (esmalte) e da faceta (dissilicato de lítio). O condicionamento interno da faceta foi feito com ácido fluorídrico (IPS Ceramic Etching Gel, IvoclarVivadent) à 5\% durante 20 segundos (Figura 9a), após essa etapa a peça foi lavada (30s) e secada (20s) totalmente com jatos de ar, o seguinte passo foi a aplicação ativa de ácido fosfórico a 37\% (Gel Etchant, Kerr) (Figura 9b) para remoção de debris de hexafluorsilicato, promovendo a limpeza da superfície interna. Seguiu-se com a aplicação de silano (Silane Primer, Kerr) (Figura 9c) por 60 segundos. A peça estará pronta para receber o adesivo e agente cimentante (Figura 9d). $\mathrm{O}$ adesivo hidrófobo (Optibond FL - Adhesive, Kerr) é então aplicado e não polimerizado, seguido pela resina composta. $\mathrm{O}$ conjunto faceta, adesivo e resina composta foi aquecido a 69 graus Celsius por 5 minutos em dispositivo próprio (Calset, Addent) enquanto o substrato dental é condicionado (Figura 10).

Quanto ao preparo do dente, após a remoção dos provisórios, fez-se a limpeza com micro-escova (Micro-escova azul, DhPro). Seguiu-se com o isolamento absoluto do campo operatório com dique de borracha (Heavy 6", Nic Tone) e grampos específicos (B4, Coltene) para então executar a microabrasão com microjato de óxido de alumínio (Aluminium Oxide $50 \mu \mathrm{m}$, Danville). O condicionamento do esmalte foi feito por meio de ácido fosfórico à 37\% (Gel Etchant, Kerr) seguido pela lavagem com água (20s) e a secagem (10s) da superfície para aplicação apenas do adesivo hidrófobo (Optibond FL - Adhesive, Kerr) visto que não havia dentina exposta. A faceta silanizada, com adesivo e resina composta aquecida foi inserida no dente, ajustada na posição correta. Os excessos da resina composta foram removidos com sonda

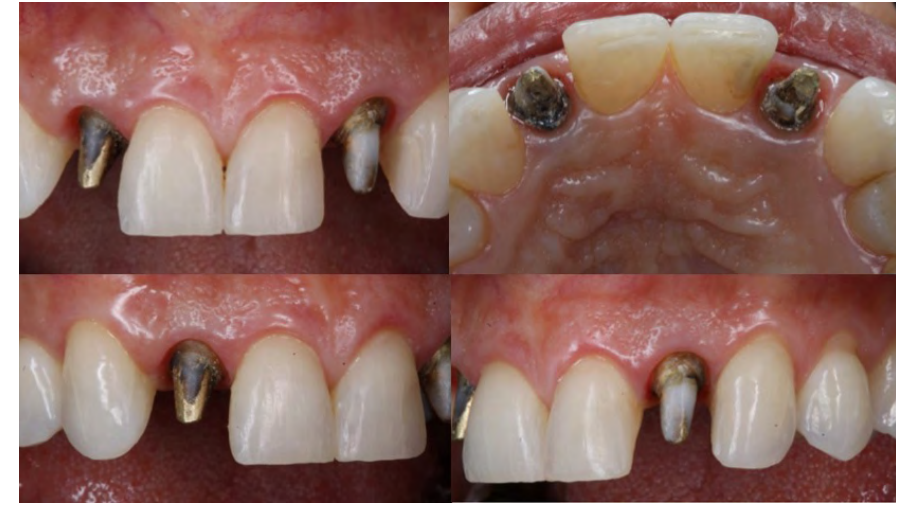

Figura 5 - Vista frontal, oclusal e lateral dos preparos dentais.

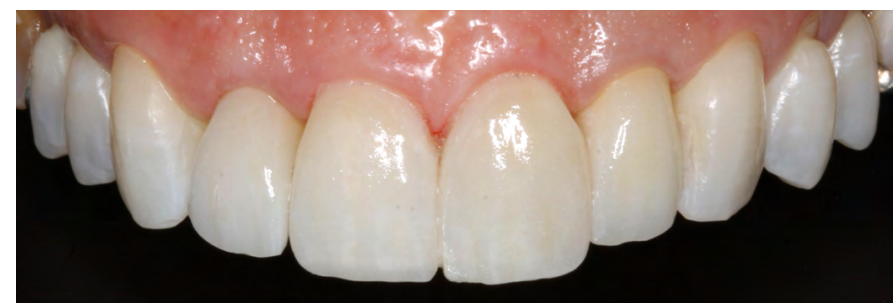

Figura 6 - Restaurações provisórias cimentadas aos preparos.

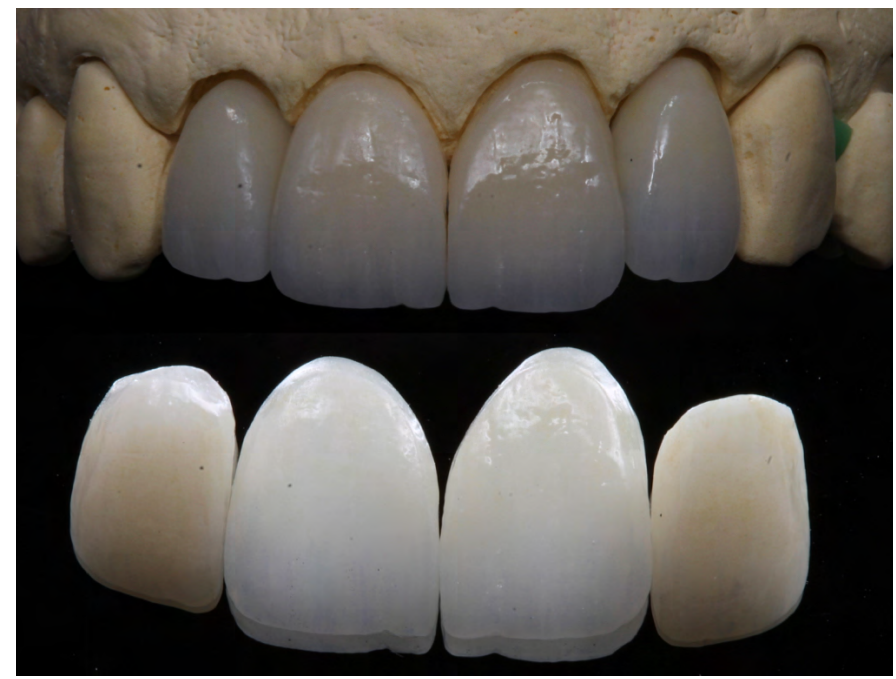

Figura 7 - Peças cerâmicas no modelo para inspeção visual de adaptação, cor e forma. Imagem mostrando a translucidez diferente entre faceta e coroa total cerâmica.

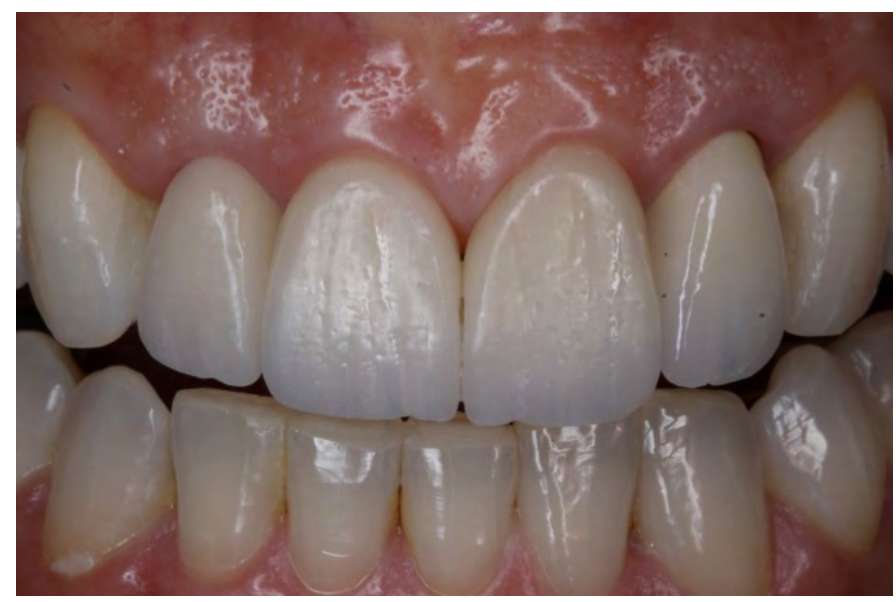

Figura 8 - Prova seca da cerâmica em boca. 
exploradora, seguindo polimerização vestibular e lingual por $2 \mathrm{x}$ 20 segundos (Valo, Ultradent) em cada (total de 80 segundos). Por fim aplicou uma camada de gel hidrossolúvel sobre as margens (para evitar a camada inibida pelo oxigênio) e foi realizada a polimerização por mais 10 segundos. Após a polimerização, os excessos de resina composta foram removidos utilizando lâmina de bisturi número 12 e lâmina beaver (Swann Morton) (Figura 11).

Logo após foram realizadas as cimentações das coroas totais cerâmicas sob isolamento relativo com afastador, rolete de algodão e fio retrator. Para evitar que o sangramento gengival contaminasse o preparo e a peça protética, aplicou-se um gel hemostático de sulfato de alumínio a 25\% (Gel-Cord, Pascal Co Inc.) seguido pela inserção de fio retrator (Siltrax EPI 00, Pascal Co Inc.). Posteriormente, a esta etapa foi feito o jateamento com

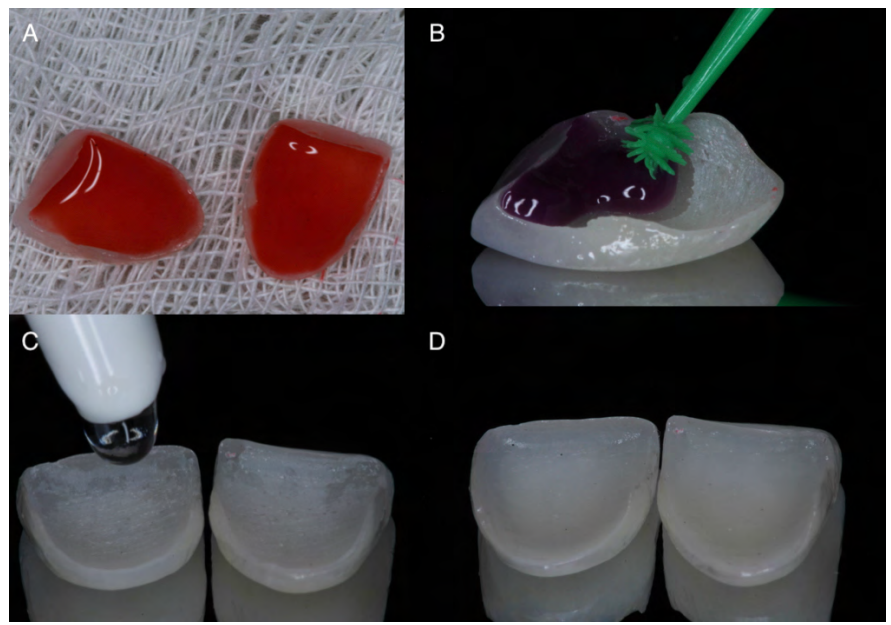

Figura 9 - Preparo da superfície cerâmica para adesão ao material cimentante. Aácido fluorídrico a 10\% sobre a peça; B- ácido fosfórico a 37\% sobre a peça; C- Silano sendo inserido na peça; D- Faceta silanizada

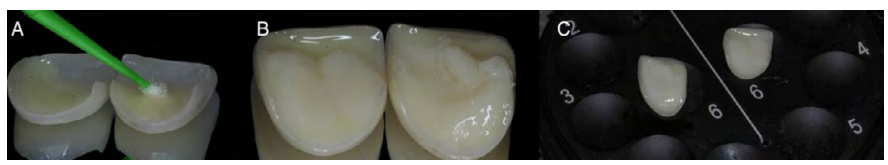

Figura 10 - Aplicação da resina composta como agente cimentante. A - aplicação do adesivo Optbond FL, B- Colocação da resina composta sobre a face interna da faceta e C- posicionamento das facetas no Calset até o momento da cimentação.

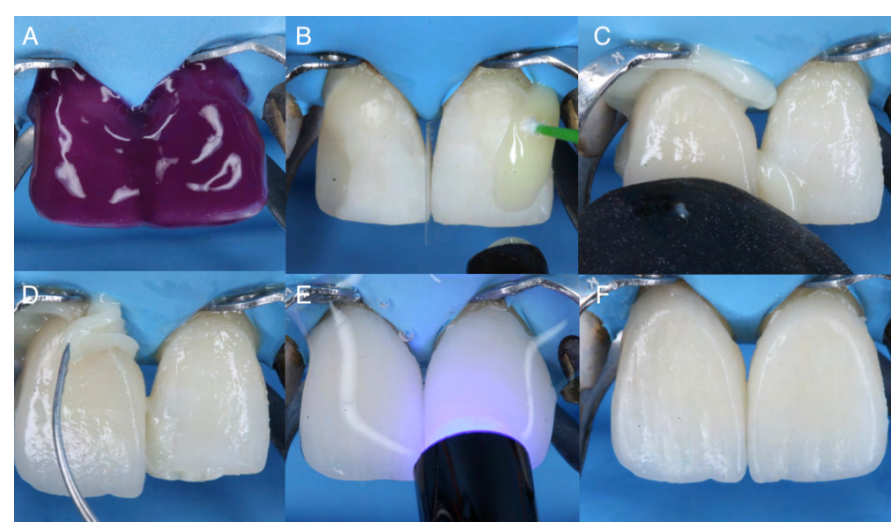

Figura 11 - Preparo da superfície do dente e cimentação da faceta. A- Aplicação do ácido fosfórico, B- Aplicação do adesivo, C- Posicionamento da faceta sobre o preparo, D- Remoção dos excessos com sonda exploradora, E- Fotopolimerização com gel hidrossolúvel após a polimerização inicial e F- Vista final pós-polimerização. óxido de alumínio (Aluminium Oxide $50 \mu \mathrm{m}$, Danville) para limpar e criar rugosidades no preparo a fim de melhorar a adesão do cimento. Condicionou-se a dentina do preparo com o mesmo ácido fosfórico a $37 \%$ por 10 segundos seguidos pela aplicação do primer por 20 segundos e adesivo por 20 segundos. A espessura do adesivo foi diminuída com jato de ar e polimerizado por 2x20 segundos, nas faces vestibular e lingual (Figura12).

Foi realizado o preparo interno da peça cerâmica com condicionamento com ácido fluorídrico a $10 \%$ durante 20 segundos (IPS Ceramic Etching Gel, IvoclarVivadent), lavagem com água, e aplicação de ácido fosfórico (Gel Etchant, Kerr) para remover os restos de ácido fluorídrico que estavam no interior da peça, que foi lavada e secada posteriormente, seguido pela aplicação de uma camada de silano (Silane Primer, Kerr) por 60 segundos na peça e secagem com ar (Figura 13). Foi feita a inserção do cimento resinoso dual (Variolink Esthetic DC Neutral, Ivoclar Vivadent) no interior da coroa e esta foi levado em posição no preparo. Removeram-se os maiores excessos com micro-escova antes de polimerizar o material cimentante. Polimerização por $3 \times 20$ segundos cada face e polimerização final de 10 segundos

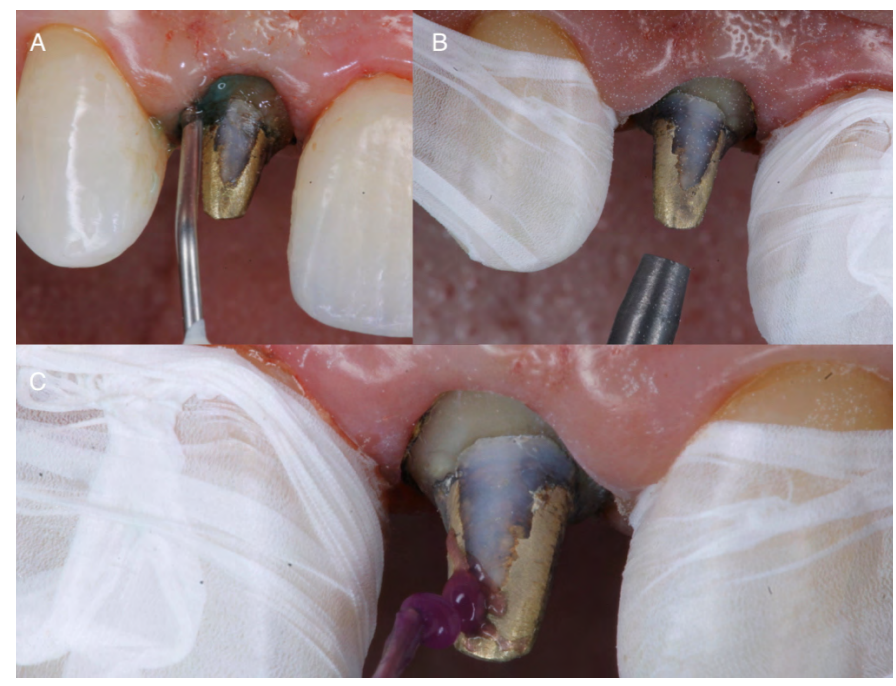

Figura 12 - Preparo das superfícies para coroa total. A- Aplicação de hemostático; B- Jateamento com óxido de alumínio; C- Aplicação de ácido fosfórico 37\%

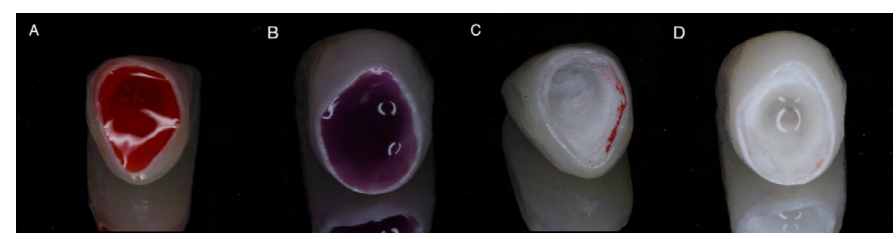

Figura 13 - A- Aplicação de ácido fluorídrico a 10\%; B- Peça protética após ser lavada e secada com remanescente de ácido fluorídrico; C- Aplicação de ácido fosfórico a 37\%; D- Aplicação de silano.

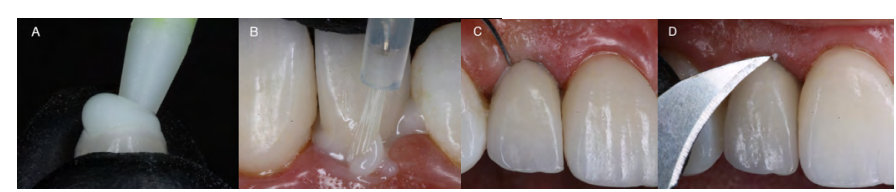

Figura 14 - A- Aplicação do cimento resinoso dual na coroa cerâmica; B- Remoção de excessos com micro-brush; C- Remoção de excessos com sonda exploradora; D- Remoção de excessos com lâmina de bisturi número 12 
com uma camada de gel hidrossolúvel (total de 140 segundos). Após a completa polimerização removeu-se os excessos com sonda exploradora, lâmina de bisturi número 12 e lâmina beaver (Swann Morton) (Figura 14 e 15). Foi feita a checagem dos contatos oclusais e a paciente retornou 15 dias após a cimentação, em consulta de Proservação, onde se observou saúde periodontal e perfeita adaptação das restaurações indiretas (Figuras 16 e 17).

\section{DISCUSSÃO}

A presença de características indesejadas ocasiona um desequilíbrio da harmonia do sorriso, prejudicando a beleza, padrão funcional, fonética e oclusão; podendo se relacionar com a redução da autoestima e bem-estar psicossocial ${ }^{3}$. Sendo assim, restabelecer a estética dentofacial de um paciente é um dos temas mais relevantes da Odontologia Restauradora ${ }^{10}$.

O presente caso clínico apresentou a indicação de diferentes técnicas restauradoras, bem como distintas técnicas de cimentação que auxiliam na longevidade da reabilitação estética.

Os fundamentos da estética se baseiam na proporção entre os dentes e no equilíbrio perfeito entre a arquitetura branca (dentes) e a arquitetura rosa (tecido gengival), tornando o sorriso harmonioso e natural. A proporção é baseada na sequência gradativa do incisivo central para o canino, denominada proporção áurea ${ }^{13}$.

A conquista dos avanços nas técnicas e materiais permite a aplicação de um conceito reabilitador minimamente invasivo e conservador da estrutura dentária. Esse conceito está sedimentado na teoria da adesão e conservação de estrutura dentária ${ }^{12}$. As cerâmicas são uma ótima opção para restaurações indiretas, pela capacidade de reproduzir a aparência dos dentes naturais e por apresentarem biomecânica semelhante ao esmalte. Além disso, apresentam resistência à compressão e desgaste, lisura de superfície, brilho e pequeno acúmulo de placa ${ }^{14,15}$.

No presente caso optou-se pelo tratamento dos quatro incisivos, visto que os laterais já apresentavam coroas provisórias de resina acrílica confeccionadas por outro cirurgião-dentista, e o dente 21 com restauração em resina composta Classe IV bastante extensa. Sendo assim, o tratamento envolvendo os elementos 11, 12, 21 e 22 foi necessário para garantir a estética (cor e forma) necessária no caso.

As diferenças na cimentação do caso se deram devido às diferentes restaurações usadas. Para a cimentação das facetas foi utilizada a técnica de Resina termomodificada (RTM), que utiliza a resina composta convencional aquecida para modificar suas propriedades reológicas e se apresentar mais fluida no momento da cimentação. As vantagens desta técnica é a utilização da resina composta que é um material restaurador e apresenta excelentes propriedades mecânicas que contribui com o aumento da resistência mecânica da restauração ${ }^{16}$. A coroa total foi cimentada com cimento resinoso dual visto a impossibilidade de fotopolimerização pela espessura da peça protética que inviabilizaria a polimerização completa do agente cimentante.

Ainda são necessários estudos comparando o protocolo de cimentação com resina composta aquecida ou cimento resinoso visando aprimorar a técnica de cimentação de restaurações cerâmicas e as suas indicações.

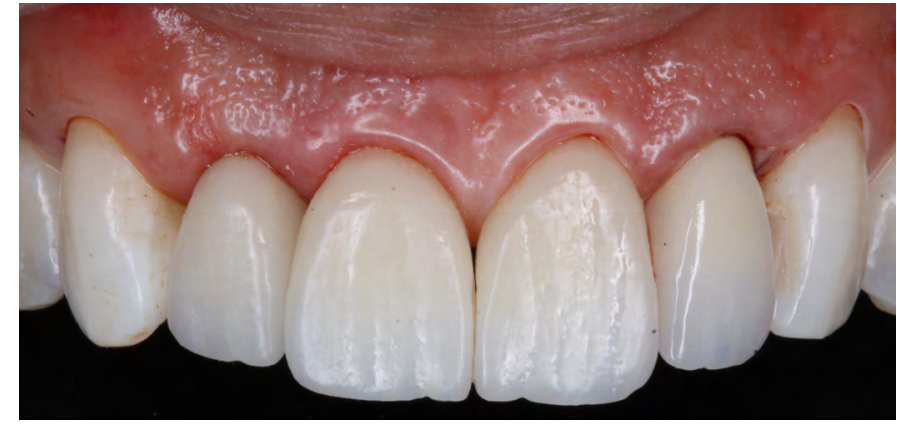

Figura 15 - Aspecto final imediato.

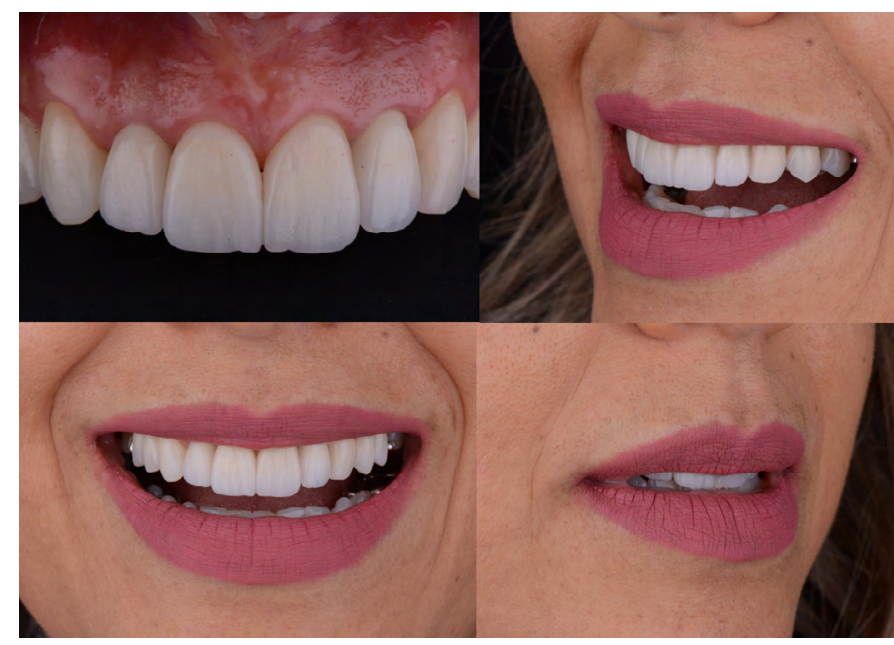

Figura 16 - Aspecto após 15 dias.

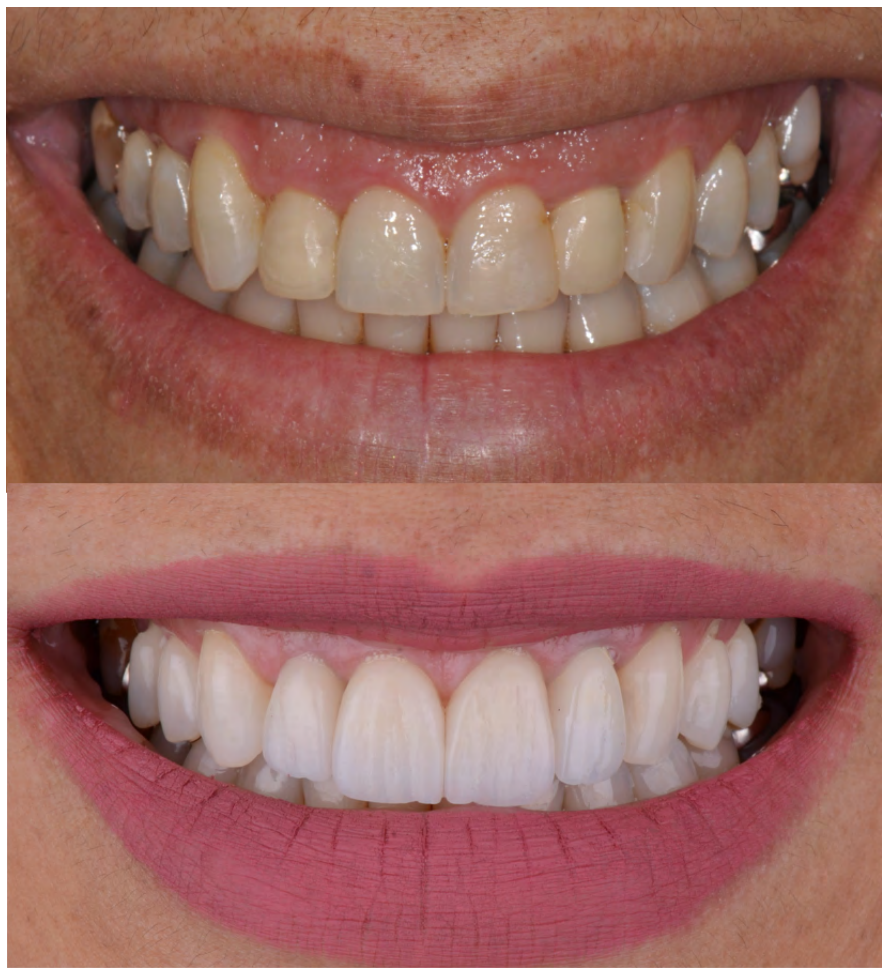

Figura 17 - Antes e depois do tratamento. 


\section{CONCLUSÃO}

As restaurações envolvendo cerâmicas odontológicas são uma ótima opção, devido sua durabilidade e sua capacidade de mimetizar o dente natural. A adesão da peça cerâmica com a estrutura dental, alta resistência à fratura, durabilidade, adaptação no terço cervical, assim como um correto planejamento e execução do caso geraram resultados clínicos satisfatórios e condizentes com os princípios estéticos do sorriso. Ao final do tratamento a paciente se demostrou satisfeita com o resultado.

\section{REFERÊNCIAS}

01. Magne P, Magne M. Treatment of extended anterior crown fractures using Type IIIA bonded porcelain restorations. J Calif Dent Assoc. 2005;33(5):387-96.

02. D'Arcangelo C, Angelis F, Vadini M, D'Amario M. Clinical evaluation on porcelain laminate veneers bonded with light-cured composite: results up to 7 years. Clin Oral Investig. 2012;16(4):1071-9.

03. Belser UC, Magne P, Magne M. Ceramic laminate veneers: continuous evolution of indications. J Esthet Dent. 1997; 9(4):197-207.

04. Benetti AR, Miranda CB, Amore R \& Pagani C. Facetas Indiretas em Porcelana: alternativa estética. J Bras Dent Estet. 2003; 2(7):186-94.

05. Mendes WP, Bonfante G, Janssen WC. Facetas Laminadas - Cerâmica e Resina: Aspectos Clínicos. In: Livro do Ano da Clínica Odontológica Brasileira. São Paulo: Ed. Artes Médicas; 2004. p. 27-59.

06. Walter RD, Raigrodski AJ. Clinical considerations for restoring mandibular incisors with porcelain laminate veneers. J Esthetic Restor Dent. 2008; 20(4): 276-81.
07. Fradeani M, Redemagni M, Corrado M. Porcelain Laminate Veneers: 6-to12-Year Clinical Evaluation-A Retrospective Study. Int J Peridontics Restorative Dentistry. 2005; 25(1): 9-17.

08. Kelly JR, Nishimura I, Campbell SD. Ceramic in dentistry: History and historical roots and current perspectives. J Prosthet Dent.1996; 75(1):18-32.

09. Aquino APT, Cardoso PC, Rodrigues MB, Takano AE, Porfírio W. Facetas de Porcelana: Solução Estética e Funcional. International Journal of Brazilian Dentistry. 2009; 5(2):142-152.

10. Souza EM, Souza JR MHS, Lopes FAM, Osternack FHR. Facetas estéticas indiretas em porcelana. JBD. 2002; 1(3):256-262.

11. Freese C, Staehle HJ, Wolff D. The assessment of dentofacial esthetics in restorative dentistry: a review of the literature. J Am Dent Assoc. 2012; 143(5):461-6.

12. Keys W, Carson SJ. Rubber dam may increase the survival time of dental restorations. Evid Based Dent. 2017; 18(1):19-20.

13. Levin EI. Dental esthetics and the Golden proportion. J Prosthet Dent. 1978; 40(3):244-52.

14. Soares PV, Zeola LF, Pereira FA, Milito GA, Machado AC. Reabilitação estética do sorriso com facetas cerâmicas reforçadas por dissilicato de lítio. Rev Odontol Bras Central. 2012; 21(58):538-43.

15. Soares PV, Faria NFB, Cardoso IO, Moura GF, Pereira AG. Abordagem multidisciplinar para reabilitação estética do sorriso com facetas cerâmicos minimamente invasivos. J Clin Dent Res. 2017; 14(1):68-88.

16. Gresnigt MMM, Özcan M, Carvalho M, Lazari P, Cune MS, Razavi P, Magne P. Effect of luting agent on the load to failure and acceleratedfatigue resistance of lithium disilicate laminate veneers. Dent Mater. 2017; 33(12):1392-1401.

\section{ABSTRACT}

Objective: This study aims to present a clinical case of anterior aesthetic rehabilitation (central and anterior superior sides), combining cemented ceramic veneers and crowns with different techniques (resin cement and heated composite resin) with the use of chemical substances as used substances. Material and Methods: Patient A.M.A., female, 47 years old, compared to the dental clinic of the Faculty of Dentistry of the Federal University of Goiás (UFG), relating dissatisfaction with the aesthetics of his smile, differences in the shape and color of his patients. On intraoral clinical examination, a provisional total crown of tooth 22 was found, and tooth 12 was poorly positioned in relation to adjacent teeth. Periodontal surgery was indicated to increase the clinical crown, tooth whitening, two ceramic veneers on the central incisors and two ceramic crowns on the upper lateral incisors. After periodontal surgery and whitening, the preparations for the full crown and preparations for the ceramic veneer were refined. The material of choice for restorations was dissolved on the site due to its great ability to mimic the natural tooth. As crowns, they were cemented with isolation relative to the operative field and double resin cement. The veneers were cemented with absolute insulation and heated composite resin. Results: After 15 days of cementation, the patient returned to the clinic where he recovered from periodontal health and perfect adaptation of the indirect restorations. Conclusion: You get an excellent aesthetic result by associating different types of restorations and adhesive cementation techniques and a patient is comfortable with different approaches and satisfaction with the aesthetic result of the treatment.

Keywords: Dental Veneers; Crowns; Dental Cements; Dental Bonding; Mouth Rehabilitation.

\author{
AUTOR PARA CORRESPONDÊNCIA \\ Priscilla Cardoso Lazari Carvalho \\ Faculdade de Odontologia do Centro Universitário de \\ Anápolis- UniEVANGÉLICA \\ Av. Universitária, Km 3,5 - Cidade Universitária - Anápolis \\ - GO, CEP: 75083-515 \\ E-mail: priscilla.lazari@docente.unievangelica.edu.br
}

